\title{
Antenna Systems for Internet of Things
}

\author{
Hassan Tariq Chattha $\left(\mathbb{D},{ }^{1}\right.$ Qammer H. Abbasi, ${ }^{2}$ Masood Ur-Rehman ${ }^{(D)}{ }^{3}$ \\ Akram Alomainy, ${ }^{4}$ and Farooq A. Tahir $\mathbb{B}^{5}$ \\ ${ }^{1}$ Department of Electrical Engineering, Faculty of Engineering, Islamic University of Madinah, Saudi Arabia \\ ${ }^{2}$ School of Engineering, University of Glasgow, Glasgow G12 8QQ, UK \\ ${ }^{3}$ School of Computer Science \& Electronic Engineering, University of Essex, Wivenhoe Park, Colchester CO4 3SQ, UK \\ ${ }^{4}$ School of Electronic Engineering and Computer Science, Queen Mary, University of London, London, E1 4NS, UK \\ ${ }^{5}$ Research Institute for Microwave and Millimeter-Wave Studies, National University of Sciences and Technology (NUST), \\ Islamabad, Pakistan \\ Correspondence should be addressed to Hassan Tariq Chattha; chattha43@hotmail.com
}

Received 2 September 2018; Accepted 2 September 2018; Published 17 September 2018

Copyright (c) 2018 Hassan Tariq Chattha et al. This is an open access article distributed under the Creative Commons Attribution License, which permits unrestricted use, distribution, and reproduction in any medium, provided the original work is properly cited.

Internet of Things (IoT) shall be imperative in incorporating different heterogeneous end systems including everything from smart homes to the industrial Internet of Things with smart agriculture, smart cities, and the smart grid. Apart from the sophisticated communication protocols, the choice of an antenna system will be a critical component of all these node end smart devices. Choosing the right antenna for an application imposes a key design challenge. As IoT modules continue to shrink incorporating more wireless technologies, making space for antennas is becoming an increasingly significant challenge. Thus, IoT-module antenna design faces the restrictions of ever-shrinking footprints while maintaining reasonable antenna performance under severe conditions such as noise, fading, and the need for efficiency. Enhanced techniques for multiplexing, interference mitigation, scheduling, and radio resource allocation work alongside the antenna design for the realization of efficient antenna systems for IoT.

The explosive growth of the Internet of Things and smart industrial applications creates many scientific and engineering challenges that are the main intentions of this special issue for ingenious research efforts for the development of efficient, cost-effective, scalable, and reliable antenna systems for IoT. The antenna designs for UWB and RFID tags in IoT enabled environment, MIMO antenna systems, transmission techniques of massive MIMO systems, and position tolerance design method and effects of randomness in element positions in antenna arrays are given particular emphasis in the contributions included in this special issue.

Contributions. This special issue contains 6 scientific papers covering a range of antenna designs, techniques, and systems related to the Internet of Things.

In "A Novel Dual Ultrawideband Cpw-Fed Printed Antenna for Internet of Things (Iot) Applications" by Q. Awais et al., the authors present a printed antenna with coplanar waveguide $(\mathrm{CPW})$ feeding having rectangular shaped blocks with dual-band characteristics. The antenna has compact dimensions of $35 \mathrm{~mm} \times 25 \mathrm{~mm}$ with an FR4 used as substrate. For increasing the impedance bandwidth and gain of the antenna, a technique of rounded corners is exploited. The two ultrawidebands covered by the proposed antenna are $1.1 \mathrm{GHz}$ to $2.7 \mathrm{GHz}$ and $3.15 \mathrm{GHz}$ to $3.65 \mathrm{GHz}$, which include $2.45 \mathrm{GHz}$ Wi-Fi/Bluetooth, majority of the $3 \mathrm{G}, 4 \mathrm{G}$ bands, and a lower $6 \mathrm{GHz} 5 \mathrm{G}$ band of $3.4 \mathrm{GHz}$ to $3.6 \mathrm{GHz}$. The simulated results are compared with the measured ones which are generally in good accordance with each other. The antenna being low-profile is a suitable candidate for the $5 \mathrm{G}$ IoT portable applications.

In "Position Tolerance Design Method for Array Antenna in Internet of Things" by C. Wang et al., the authors discuss the position error of array antenna which is a significant factor of deteriorating the gain and sidelobe of the array, thus seriously affecting the performance of antenna array for 
the Internet of Things (IoT). Using the sensitivity analysis theory, the authors derive the sensitivity of the array radiation field with respect to the position of the antenna element. The authors have also proposed a novel design method of position tolerance for array antenna and have applied it to a $20 \times 20$ planar array and have compared it with the antenna array designed by the traditional method. It is found that the gain loss of both of the antenna array is the same, i.e., $0.5 \mathrm{~dB}$, whereas the peak side lobe level is lowered by 1.937 $\mathrm{dB}\left(\phi=0^{\circ}\right) / 1.586 \mathrm{~dB}\left(\phi=90^{\circ}\right)$. In addition, the uncertainty analysis is also performed which has proved that the newly designed array has higher probability to obtain the required performance characteristics, which validates the new design method being innovative and effective.

In "On the Capacity and Transmission Techniques of Massive MIMO Systems," the author M. A. Haleem discusses the Massive-MIMO wireless system which is a multiuser system having base stations consisting of a large number of antenna elements with respect to the number of user devices with each equipped with a single antenna. The potential of the massive MIMO system is its ability to realize multiuser channels with near zero mutual coupling. In a high bit rate massive MIMO system with $\mathrm{m}$ base station antennas and $\mathrm{n}$ users, downlink capacity increases as $\log _{2} \mathrm{~m} \mathrm{bps} / \mathrm{Hz}$ and the capacity per user decreases as $\log _{2} \mathrm{n}$ bps/Hz. This capacity can be obtained by power sharing using signal weighting vectors aligned with respective $1 \mathrm{x} \mathrm{m}$ channels of the users. For a low bit-rate transmission, time sharing obtains the capacity equivalent to that of power sharing. If the channel coupling factor increases, the system capacity decreases. The probability distribution of channel coupling factor is a convenient tool to predict the number of antennas needed to qualify a system as Massive MIMO.

In "Tunable Platform Tolerant Antenna Design for RFID and IoT Applications Using Characteristic Mode Analysis" by A. Sharif et al., the authors highlight the significance of Radio Frequency Identification (RFID) as an emerging technology for sensing, identifying, tracking, and localizing different goods as being cost effective in tagging a large number of goods. The authors have proposed a platform tolerant RFID tag antenna having tuning capabilities. The Characteristic Mode Analysis (CMA) is used to design and optimize the proposed tag antenna. The proposed antenna contains a patch folded around an FR4 substrate and a loop as feeder printed on a paper substrate. Feeding loop is stacked over the folded patch thus providing impedance matching with different RFID chips. Having separate radiator and feeder, the proposed antenna exhibits the capability of getting its impedance matched with a variety of RFID chips. The frequency band covered by the proposed tag antenna is an American RFID band of 902-928 MHz and has the capability of being able to be tuned to the European RFID band of $865-868 \mathrm{MHz}$ ) using tunable strips. For the demonstration of platform tolerant operation, the RFID tag is mounted on different materials to measure its maximum read range, which is found to be $4.5 \mathrm{~m}$ in free space, on a dielectric, and a value of $6.5 \mathrm{~m}$ when placed above a $200 \mathrm{~mm} \times 200 \mathrm{~mm}$ metal plate.

In "Compact Ultra-Wide Band MIMO Antenna System for Lower 5G Bands" by H. Al-Saif et al., the authors propose a planar antenna with ultrawideband characteristics for MIMO applications. The presented antenna is simulated in the CST microwave studio and is designed to cover lower $5 \mathrm{G}$ bands from 2 to $12 \mathrm{GHz}$, thus covering the IEEE $802.11 \mathrm{a} / \mathrm{b} / \mathrm{g} / \mathrm{n} / \mathrm{ac}$ as well. The proposed antenna has compact and miniaturized dimensions of $25 \mathrm{~mm} \times 13 \mathrm{~mm} \times 0.254 \mathrm{~mm}$. The measured and simulated results are presented having good accordance with each other. The simulated and measured reflection coefficients $S_{11}$ for the proposed design are below $-10 \mathrm{~dB}$ over the frequency band in interest. The mutual coupling found between the two antennas is under $-20 \mathrm{~dB}$ in desired frequency range. The $2 \mathrm{D}$ radiation patterns in $\mathrm{E}$ and $\mathrm{H}$ planes exhibit omnidirectional far fields with a peak measured gain of $4.8 \mathrm{~dB}$. The fractional bandwidth of the proposed antenna is found to be around $143 \%$, thus showing its ultrawideband characteristics.

In "Effect of Randomness in Element Position on Performance of Communication Array Antennas in Internet of Things" by C. Wang et al., the authors discuss the active phased array antennas, being a critical component of wireless communication, and face the restrictions of creating effective performance with the effect of randomness in the position of the array element, which are inevitably produced in the manufacturing and operating processes of the antenna. A new method for efficiently and effectively evaluating the statistic performance of the antenna is presented, with consideration of randomness in element position. A coupled structuralelectromagnetic statistic model for array antenna is proposed from the viewpoint of electromechanical coupling. In addition, a $12 \times 12$ planar array is illustrated to evaluate the performance of the antenna with the saddle-shaped distortion and random position error. The results show that the presented model can obtain the antenna performance quickly and effectively, providing an advantageous guidance for structural design and performance optimization for array antennas in a wireless application.

These contributions bring a good insight of the present development activities and research happening related to antenna systems for the Internet of Things. The editors are optimistic that this special issue will contribute to the knowledge base and benefit the scientific community.

\section{Conflicts of Interest}

Authors have no conflicts of interest.

\section{Acknowledgments}

The editors would like to commend the contributions of the authors for making the special issue a success. Taking this opportunity, the editors would like to appreciate the efforts of the reviewers as well for the rigorous review process, thus enhancing the quality of the manuscripts submitted.

\section{Hassan Tariq Chattha \\ Qammer H. Abbasi \\ Masood Ur-Rehman \\ Akram Alomainy \\ Farooq A. Tahir}




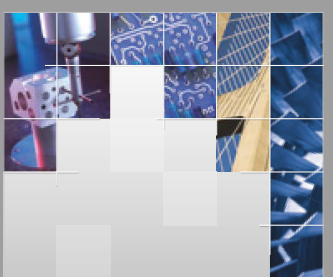

\section{Enfincering}
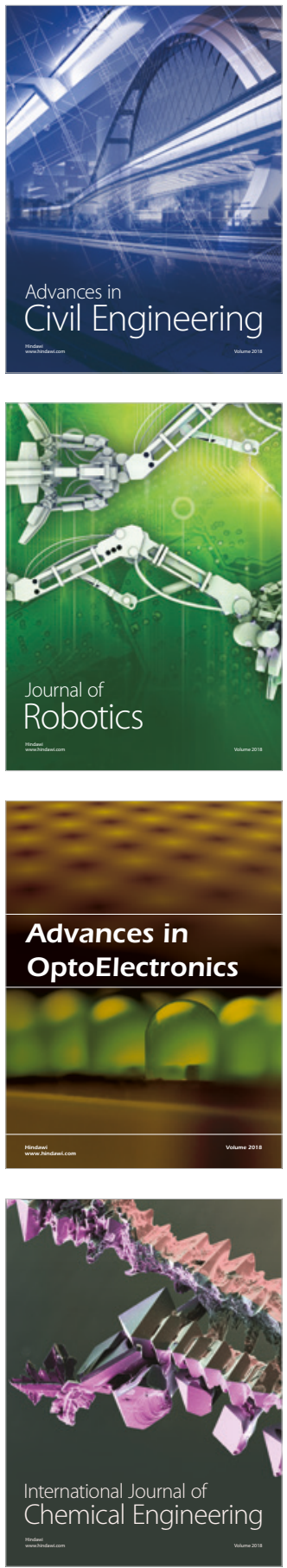

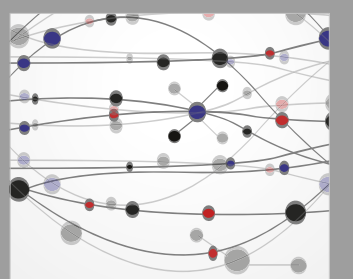

\section{Rotating \\ Machinery}

The Scientific World Journal

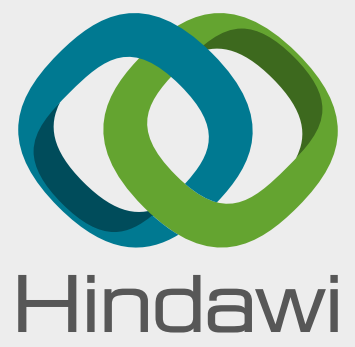

Submit your manuscripts at

www.hindawi.com
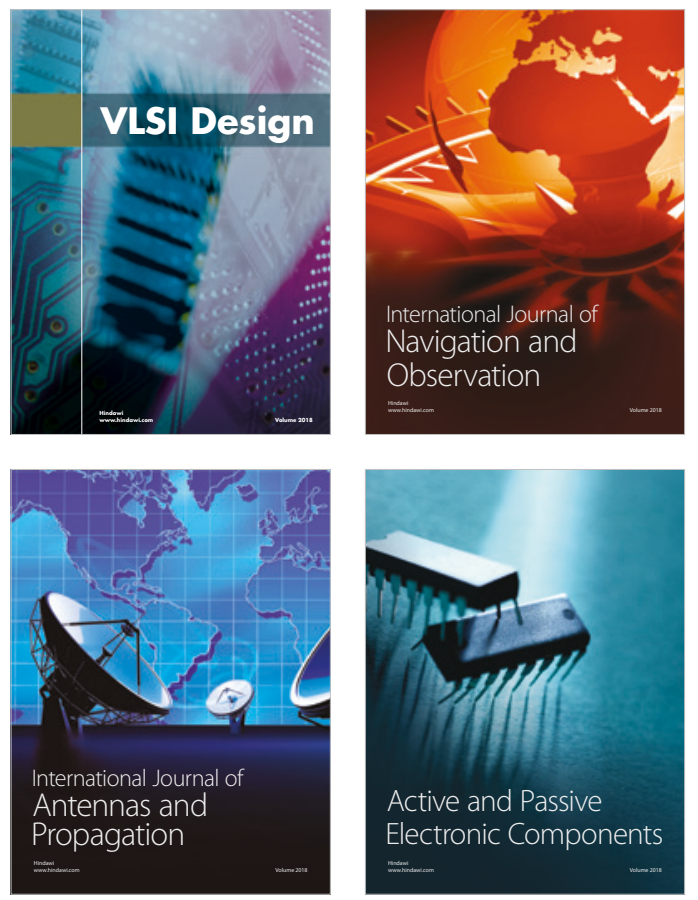
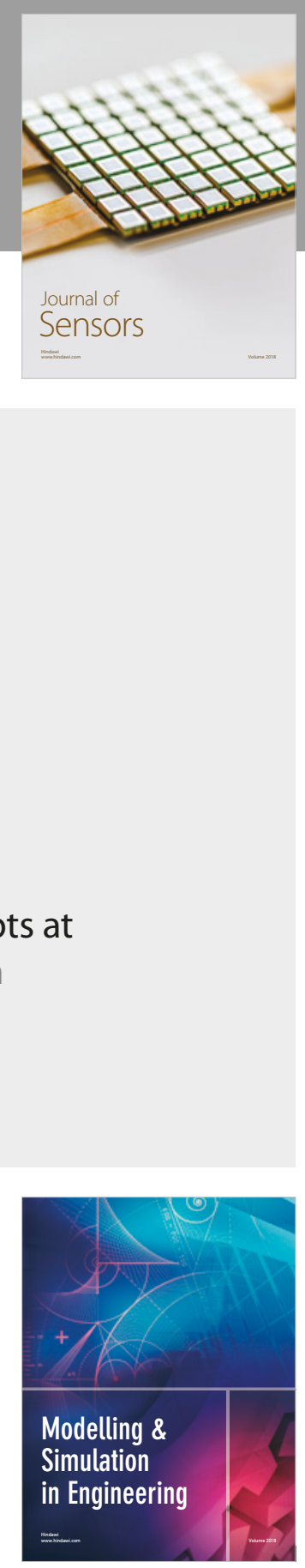

\section{Advances \\ Multimedia}
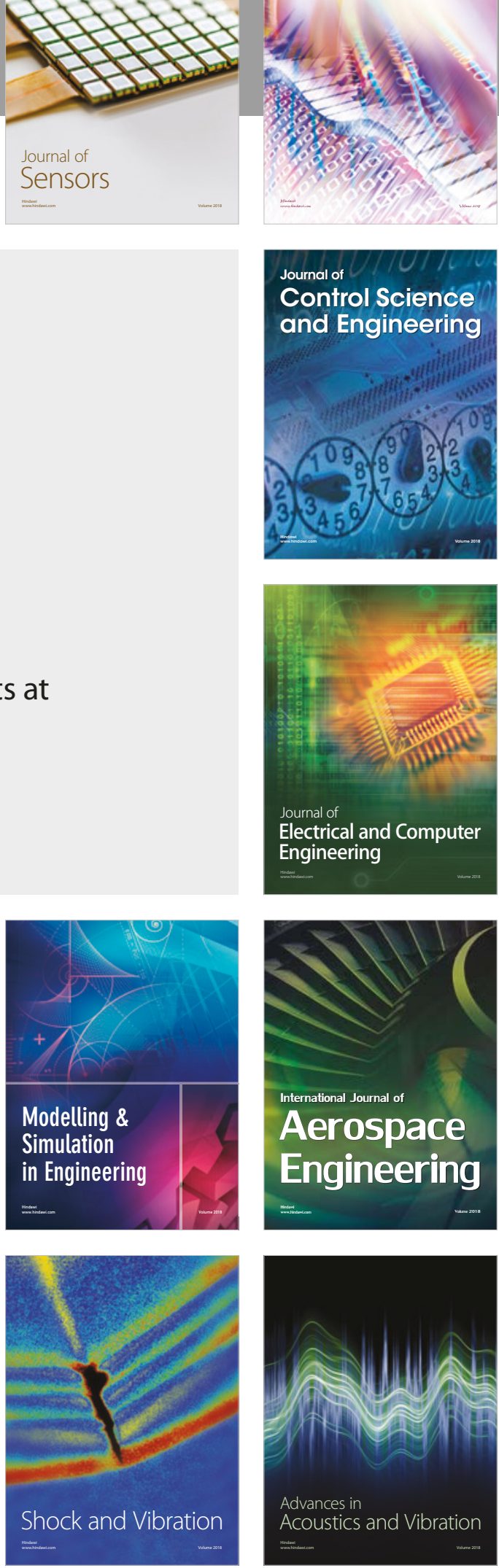